\title{
Uso do sensoriamento remoto na estimativa do índice de área foliar em floresta tropical
}

\author{
Use of remote sensing in estimating leaf area index in tropical Forest
}

Victor Hugo de Morais Danelichen', Marcelo Sacardi Biudes², Nadja Gomes Machado ${ }^{3}$, Maísa Caldas Souza Velasque ${ }^{2}$, Bernardo Barbosa da Silva ${ }^{4}$ e José de Souza Nogueira ${ }^{2}$

\author{
${ }^{1}$ Universidade de Cuiabá, MT, Brasil \\ danelichen@fisica.ufmt.br \\ ${ }^{2}$ Universidade Federal de Mato Grosso, MT, Brasil \\ marcelo@fisica.ufmt.br; maisacaldas@fisica.ufmt.br; nogueira@ufmt.br \\ ${ }^{3}$ Instituto Federal de Mato Grosso, MT, Brasil \\ nadja.machado@blv.ifmt.edu.br \\ ${ }^{4}$ Universidade Federal de Pernambuco, PE, Brasil \\ bbdasilva.ufpe@gmail.com
}

\begin{abstract}
Resumo
A estimativa do índice de área foliar (IAF) em ecossistemas florestais é realizada, em sua maioria, por métodos que incluem a intensidade da radiação eletromagnética, transmitida através do dossel. No entanto, essas técnicas não permitem a representação espacial do IAF. Sendo assim, o objetivo deste trabalho foi avaliar o IAF de uma floresta de transição Amazônia-Cerrado estimado por dados de sensores orbitais. Para tanto, o IAF estimado pelo METRIC (Mapping EvapoTranspiration with high Resolution and Internalized Calibration) com imagens TM - Landsat 5 e pelo produto MOD15A2 do MODIS (Moderate Resolution Imaging Spectroradiometer) foi validado com medidas locais por interceptação de luz em uma torre micrometeorológica em 2008. Mapas de distribuição espacial do IAF foram preparadas para os oito dias considerados. O IAF estimado pelo produto MOD15A2 apresentou concordância com as medidas em campo, enquanto que o METRIC não se mostrou capaz de produzir valores do IAF similares àqueles obtidos em campo. Mesmo que o METRIC apresentou limitações no seu uso, trabalhos futuros são necessários para parametrizar a sua equação para uma maior série espaço/temporal de dados.
\end{abstract}

Palavras-chave: Floresta tropical; Imagens de satélite; Função do ecossistema; Microclima.

\begin{abstract}
The estimation of leaf area index (LAI) in forest ecosystems is generally performed by methods that include the intensity of electromagnetic radiation transmitted through the canopy. However, these techniques do not allow for the spatial LAI representation. Thus, the aim of this study was to evaluate the LAI of an Amazon-Cerrado transition forest estimated by data from satellite sensors. Thus, the LAI estimated by METRIC (Mapping Evapotranspiration with high Resolution and internalized Calibration) with TM - Landsat 5 imagery and the product MOD15A2 of MODIS (Moderate Resolution Imaging Spectroradiometer) was validated with local light interception measurements of a micrometeorological tower in 2008. Spatial distribution LAI were prepared for eight days considered. The LAI estimated by the MOD15A2 product agree with measurements in the field, while the METRIC was not able to produce LAI values similar to those obtained in the field. Even if the METRIC presented limitations in their use, further work is needed to parameterize your equation for a greater spatial/ temporal range data.
\end{abstract}

Keywords: Tropical forest; Satellite images; Ecosystem function; Microclimate. 


\section{Introdução.}

Nos últimos anos, o sensoriamento remoto tem recebido maior atenção, pois possibilita o monitoramento de diversos fenômenos meteorológicos e ambientais, oferecendo suporte às previsões de tempo e ao melhor entendimento das mudanças climáticas com vista à preservação ambiental. Como consequência, tem-se tornado uma ferramenta poderosa para a obtenção de informações necessárias ao manejo, gerenciamento e gestão de recursos naturais, como água, solo e vegetação (ALLEN et al., 2007; CROSMAN \& HOREL, 2009).

Os dados provenientes de sensores orbitais possibilitam o estudo do comportamento dinâmico da vegetação. Tal abordagem procura tornar mais operacional o sistema de monitoramento da vegetação, principalmente de extensas áreas, por meio das refletâncias da superfície vegetada em bandas espectrais do visível e infravermelha próximo coletada a bordo de satélites ambientais (ALLEN et al., 2007). Além disso, diferentes atributos biofísicos e bioquímicos do dossel também podem ser avaliados por produtos derivados de combinações das diversas bandas espectrais do sensor MODIS (Moderate Resolution Imaging Spectroradiometer), a bordo dos satélites Terra e Aqua (CROSMAN \& HOREL, 2009).

O índice de área foliar (IAF) é definido como a razão entre a área total de todas as folhas por unidade de área da ocupada pela vegetação. $\mathrm{O}$ mesmo pode ser estimado localmente segundo diferentes métodos, mas também por meio de equações que combinam as refletâncias medidas pelos sensores orbitais, a exemplo dos sensores Thematic Mapper (TM) e MODIS, a bordo dos satélites Landsat 5 e Terra/ Aqua, respectivamente. Esse índice é um componente importante de modelos destinado a estudo dos ciclos biogeoquímicos de um ecossistema, por determinar o microclima abaixo e acima do dossel, controlar a interceptação da água, extinção da radiação e as trocas gasosas de água e carbono (Resende et al., 2010; Biudes et al., 2014a).

As estimativas de IAF provenientes de dados de sensores orbitais não podem ser generalizadas, necessitando da validação de suas estimativas e produtos em situações específicas (PRIVETTE et al., 2002). O algoritmo do IAF referente ao produto MOD15A2 se baseia em seis tipos de cobertura arquitetônica: gramíneas e cereais; arbustos; culturas folhosas, savanas, florestas latifoliadas; agulha e florestas de folha (MYNENI et al., 2002). Outros modelos, como o METRIC (Mapping Evapo Transpiration with high Resolution and Internalized Calibration) desenvolvido por Allen et al. (2007) foram obtidos empiricamente com dados de campo de culturas agrícolas, cuja heterogeneidade é menor que as de florestas.

A validação do IAF obtido com base em medições realizados por sensoriamento remoto pode ser realizada por medidas realizadas diretamente no campo. Para tanto, existem diferentes técnicas de obtenção do IAF em condições de campo (BRÉD A, 2003). Os métodos diretos necessitam da retirada de amostra de folhas, modificando o ambiente de estudo (NACKAERTS et al., 2000), enquanto que os métodos indiretos baseiam-se na medida da interceptação da luz, não modificando o ambiente de estudo e podendo, ainda, ser realizado ao longo de um grande período de tempo, por sua maior operacionalidade (BRÉD A, 2003).

Métodos baseados em processos envolvendo ecossistemas são muitas vezes necessários para produzir análises quantitativas do comportamento dinâmico da vegetação e o IAF é um parâmetro chave de entrada para tais simulações. Ecofisiologistas, agricultores e silvicultores, em muitas de suas aplicações, requerem informações sobre o índice de área foliar do dossel. No entanto, a relação entre ecossistemas e a atmosfera é de difícil quantificação, devido à variabilidade espacial e temporal, como ciclos anuais e variabilidade interanual relacionado com a cultura, estratificação e heterogeneidade.

Desde as revisões e trabalhos de Welles (1990) e Bréda (2003), muitas comparações entre os métodos diretos e indiretos de medição de IAF foram publicadas para diferentes culturas e povoamentos florestais (GITELSON et al., 2007; JUPP et al., 2008; BIUDES et al., 2014a).

Apesar do uso sistemático de sensores que medem o IAF diretamente por técnicas de interceptação de luz, novos tópicos, incluindo a análise de erro, calibração cruzada, estratégia de amostragem, validação de escala espacial ou técnicas de sensoriamento remoto aplicado a estudos de ecossistemas estão surgindo a partir da literatura recente. Sendo assim, o objetivo deste trabalho foi avaliar o IAF de uma floresta de transição Amazônia-Cerrado estimado por dados de sensores orbitais.

\section{Material e Métodos}

Este estudo foi desenvolvido em uma área plana localizada a aproximadamente $60 \mathrm{~km}$ NE de Sinop,

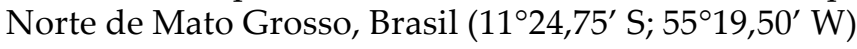
(Figura 1). O solo é caracterizado como NEOSSOLO QUARTZARÊNICO Órtico Típico A moderado álico, com profundidade de $50 \mathrm{~cm}$ e textura arenosa com $83,6 \%$ de areia, $4,4 \%$ de silte e $2,2 \%$ de argila (PRIANTE FILHO et al., 2004). Este solo tem alta porosidade e drena rapidamente as chuvas. A vegetação consiste de espécies perenes características de floresta de transição em Mato Grosso, tais como Qualea sp., Vochysia sp., Ocotea sp e Mezilaurus itauba, com alta diversidade, não havendo predominância de uma única espécie de árvore (Vourlitis et al., 2011).

O clima da região é do tipo tropical quente e úmido (Aw, segundo classificação de Köppen). Este tipo climático, predominante do centro-norte do Estado, é caracterizado pela presença de duas estações bem definidas: uma chuvosa, no período de outubro a abril, e outra seca, de maio a setembro. A temperatura média 
anual da área de estudo é $24^{\circ} \mathrm{C}$, e a precipitação média anual de $2000 \mathrm{~mm}$. As características climatológicas da floresta de transição são similares às da floresta tropical e do cerrado. Entretanto, a região de floresta de transição recebe aproximadamente $200 \mathrm{~mm}$ a menos de precipitação anual em relação à floresta tropical, e $500 \mathrm{~mm}$ a mais que o cerrado (BIUDES et al., 2015).

Os dados de radiação fotossinteticamente ativa foram coletados entre janeiro e outubro de 2008 por meio de transdutores de densidade de fluxo fótico na faixa de comprimento de onda de 400 a $700 \mathrm{~nm}$, modelo LI-190SB (LI-COR, Lincoln, Nebraska, USA), instalados acima (40 m, sendo um sensor para radiação incidente e outro para a refletida) e um abaixo do dossel da floresta ( $1 \mathrm{~m}$, somente para radiação incidente). Os dados foram registrados a cada 30 minutos em um sistema de aquisição de dados, modelo CR 10X (Campbel, Logan, UT, USA).

A fração de radiação interceptada foi calculada pelo método de Monsi e Saeki (1953). Os dados instantâneos da radiação fotossinteticamente ativa acima e abaixo do

$$
f_{c}=\frac{I_{o}}{\left(I-I_{r}\right)}
$$

dossel foram integrados diariamente e utilizados em (1).

Em que $f c$ é fração de radiação interceptada, I é a radiação fotossinteticamente ativa diária incidente no topo do dossel ( $\left.\mathrm{mol} \mathrm{m}^{-2} \mathrm{dia}^{-1}\right)$, Ir é a radiação fotossinteticamente ativa diária refletida pelo dossel $\left(\mathrm{mol} \mathrm{m}^{-2}\right.$ dia $^{-1}$ ) e Io é radiação fotossinteticamente ativa diária que

$$
I A F=\frac{\ln \left(1-f_{c}\right)}{-k}
$$

atinge a superfície $\left(\mathrm{mol} \mathrm{m} \mathrm{m}^{-2} \mathrm{dia}^{-1}\right)$.

O índice de área foliar foi estimado por (2).
Em que IAF é o índice de área foliar $\left(\mathrm{m}^{2} \mathrm{~m}^{-2}\right)$, fc é a fração de radiação interceptada e $\mathrm{k}$ é o coeficiente de extinção do dossel $\left(\mathrm{m}^{2} \mathrm{~m}^{-2}\right)$, estimado a partir do ângulo de elevação do Sol, de acordo com o método proposto por Goudriaan (1988).

Neste trabalho foram utilizados dois métodos de estimativa do IAF por dados de sensores orbitais. O primeiro foi o produto IAF do MODIS (MOD15A2), o qual tem uma resolução espacial de $1 \mathrm{~km}$ em uma grade senoidal, com frequência de 8 dias de disponibilidade de informações (GITELSON et al., 2007). O algoritmo do MODIS para o IAF é baseado na teoria de transferência radioativa tridimensional e desenvolvido por inversão usando uma abordagem de tabela de pesquisa.

O produto utilizado neste estudo (MOD15A2 - collection 5) é produzido e distribuído ao público pelo Centro de Dados EROS do Active Archive Center (EDC DAAC, disponível em http://daac.ornl.gov/cgi-bin/MODIS/GR_ col5_1/mod_viz.html). Neste trabalho foram analisados os dados do MOD15A2 com as coordenadas $-11,41^{\circ} \mathrm{S}$ e $-55,32^{\circ}$ O para a floresta de transição Amazônia-Cerrado que inclui a torre micrometeorológica. $\mathrm{O}$ valor do IAF de cada célula foi analisado e avaliado quanto à qualidade (QA), que determinou o tipo de algoritmo utilizado ou a inexistência de dados.

O outro método de estimativa do IAF por meio de sensores orbitais (METRIC) foi baseado no trabalho de Allen et al. (2007). Para tanto, foram utilizadas oito imagens para a floresta de transição Amazônia-Cerrado (Sinop) correspondentes ao ano de 2008, captadas pelo sensor TM - Landsat 5, adquiridas junto à Divisão de Geração de Imagens do Instituto Nacional de Pesquisas Espaciais (INPE). Essas imagens correspondem à órbita 226 e ponto 68 cobrindo a floresta de transição Amazônia-Cerrado, cujas informações constam na Tabela 1.

Tabela 1 - Data de captação das Imagens (DCI) pelo TM - Landsat 5, dia sequencial do ano (DSA), horário local da captação (hora), quadrado da distância relativa Terra-Sol (dr), elevação solar (E) e ângulo zenital (Z)

\begin{tabular}{c|c|c|c|c|c}
\hline DCI & \multicolumn{1}{c}{ DSA } & hora & dr & \multicolumn{2}{c}{ E } \\
\hline $09 / 02 / 08$ & 041 & $09: 35$ & 1,329 & 56,90 & 33,10 \\
\hline $15 / 05 / 08$ & 136 & $09: 32$ & 1,032 & 47,54 & 42,46 \\
\hline $16 / 06 / 08$ & 168 & $09: 32$ & 1,032 & 43,55 & 46,45 \\
\hline $02 / 07 / 08$ & 184 & $09: 31$ & 1,032 & 43,17 & 46,83 \\
\hline $18 / 07 / 08$ & 200 & $09: 31$ & 1,032 & 44,11 & 45,89 \\
\hline $03 / 08 / 08$ & 216 & $09: 30$ & 1,032 & 46,34 & 43,66 \\
\hline $19 / 08 / 08$ & 231 & $09: 30$ & 1,032 & 49,63 & 40,37 \\
\hline $09 / 12 / 08$ & 344 & $09: 28$ & 1,032 & 59,29 & 30,71 \\
\hline
\end{tabular}


A calibração radiométrica (MARKHAM \& BAKER, 1987) das imagens foi realizada por (3) pela conversão do número digital (ND) em radiância espectral monocromática das bandas refletivas do Landsat $5-\mathrm{TM}$ (canais 1, 2, 3, 4, 5 e 7).

$$
L_{\lambda i}=a_{i}+\left(\frac{b_{i}-a_{i}}{255}\right) N D
$$

Em que a e b são as radiâncias espectrais mínima e máxima $\left(\mathrm{W} \mathrm{m}^{-2} \mathrm{sr}^{-1} \mu \mathrm{m}^{-1}\right)$, presentes na Tabela 2 ei corresponde as bandas $(1,2,3,4,5,6$ e 7) do satélite Landsat 5 - TM.

Tabela 2 - Descrição dos canais do TM - Landsat 5 (CHANDER et al., 2007), com os correspondentes intervalos de comprimento de onda $(\lambda)$, coeficientes de calibração para radiâncias mínima (a) e máxima (b) em W m ${ }^{-2} \mathrm{sr}^{-1} \mu \mathrm{m}^{-1}$ e irradiâncias espectrais no topo da atmosfera $\left(\mathrm{k} \lambda \mathrm{i} ; \mathrm{W} \mathrm{m}^{-2} \mu^{-1}\right)$

\begin{tabular}{c|c|c|c|c}
\hline Bandas & $\lambda(\mu \mathrm{m})$ & $\mathrm{a}$ & $\mathrm{b}$ & $\mathrm{k} \lambda \mathrm{i}$ \\
\hline 1 (azul) & $0,45-0,52$ & $-1,52$ & 193,0 & 1957 \\
\hline 2 (verde) & $0,52-0,60$ & $-2,84$ & 365,0 & 1826 \\
\hline 3 (vermelho) & $0,63-0,69$ & $-1,17$ & 264,0 & 1554 \\
\hline 4 (IV-próx.) & $0,76-0,79$ & $-1,51$ & 221,0 & 1036 \\
\hline 5 (IV-médio) & $1,55-1,75$ & $-0,37$ & 30,2 & 215,0 \\
\hline 6 (IV-termal) & $10,4-12,5$ & 1,2378 & 15,303 & - \\
\hline 7 (IV-médio) & $2,08-2,35$ & $-0,15$ & 16,5 & 80,67 \\
\hline
\end{tabular}

A refletância monocromática planetária de cada banda (@入i), definida pela razão entre a integração hemisférica da radiância monocromática e a irradiância solar monocromática incidente em cada pixel, foi obtida por (4).

$$
\rho_{\lambda i}=\frac{\pi \cdot L_{\lambda i}}{K_{\lambda i} \cdot \operatorname{Cos} Z \cdot d_{r}}
$$

Em que $L \lambda i$ é a radiância espectral de cada banda, $\mathrm{k} \lambda \mathrm{i}$ é a irradiância solar espectral de cada banda no topo da atmosfera ( $\mathrm{W} \mathrm{m}^{-2} \mu \mathrm{m}^{-1}$, Tabela 2$), \mathrm{Z}$ é o ângulo zenital solar e dr é o quadrado da razão entre a distância média Terra-Sol (ro) e a distância Terra-Sol (r) em cada dia sequencial do ano (DSA), calculado por (5) (IQB AL, 1983).

$$
d_{r}=1+0,033 \cos (D S A .2 \pi / 365)
$$

O Índice de Vegetação Ajustado para os Efeitos do Solo (Soil Adjusted Vegetation Index - SAVI) é um índice que busca amenizar os efeitos do "background" do solo e é um indicador da biomassa (HUETE, 1998) que pode ser obtido por equações empíricas com bons resultados. Esse índice procura amenizar os efeitos da refletância do solo e foi calculado por (6) (HUETE, 1998).

$$
S A V I=\frac{(1+L)\left(\rho_{\lambda 4}-\rho_{\lambda 3}\right)}{\left(L+\rho_{\lambda 4}+\rho_{\lambda 3}\right)}
$$

Em que o fator L é função do tipo de solo da área de estudo e sua determinação requer um conhecimento a priori da resposta espectral do solo. O valor desse fator é crítico na minimização dos efeitos das propriedades ópticas do solo na refletância da vegetação e em muitas aplicações tem sido usado um valor de $L=0,1$ para variações de primeira ordem na imagem, como sendo um valor otimizado dessa refletância (HUETE, 1998).

O IAF pelo METRIC foi computado por (7) (ALLEN et al., 2007).

$$
I A F=-\frac{\ln \left(\frac{0,69-S A V I}{0,59}\right)}{0,91}
$$

Para analisar a estimativa do IAF pelo produto MODIS e pelo METRIC foram utilizados a raiz do erro quadrado médio (REQM), o teste $t$ para amostras independentes ( $p$-valor $<0,05$ ) e a correlação de Pearson ( $p$-valor $<0,05$ ).

$$
R E Q M=\left(\frac{\sum_{i=1}^{N}\left(I A F_{e s t}-I A F_{m e d}\right)^{2}}{N}\right)
$$

\section{Resultados e Discussão}

A seguir são apresentados os resultados e a discussão concernente às variabilidades de parâmetros biofísicos da superfície obtida em um alvo no norte de Mato Grosso nas proximidades do município de Sinop (Figura 1). Na Tabela 3 constam os respectivos valores do Índice de Área Foliar (IAF) medido em campo pela interceptação de luz e Índice de Vegetação Ajustado para os Efeitos de Solo (SAVI) e o IAF pelo METRIC obtidos a partir das imagens TM - Landsat 5 e o IAF pelo produto MODIS.

Tabela 3 - Valores Índice de Vegetação Ajustado para os Efeitos do Solo (SAVI) e IAF pelo METRIC, produto MODIS e medido em campo

\begin{tabular}{c|c|c|c|c}
\hline DSA & SAVI & $\begin{array}{c}\text { IAF } \\
\text { METRIC }\end{array}$ & $\begin{array}{c}\text { IAF } \\
\text { MODIS }\end{array}$ & $\begin{array}{c}\text { IAF } \\
\text { Med. }\end{array}$ \\
\hline $09 / 02 / 08$ & 0,52 & 1,4 & 4,2 & 8,5 \\
\hline $15 / 05 / 08$ & 0,58 & 1,8 & 5,4 & 7,4 \\
\hline $16 / 06 / 08$ & 0,54 & 1,5 & 5,2 & 6,4 \\
\hline $02 / 07 / 08$ & 0,62 & 2,3 & 5,5 & 6,0 \\
\hline $18 / 07 / 08$ & 0,62 & 2,3 & 5,9 & 8,1 \\
\hline $03 / 08 / 08$ & 0,63 & 2,4 & 5,9 & 7,4 \\
\hline $19 / 08 / 08$ & 0,64 & 2,6 & 6,0 & - \\
\hline $09 / 12 / 08$ & 0,62 & 2,4 & - & - \\
\hline & & & &
\end{tabular}




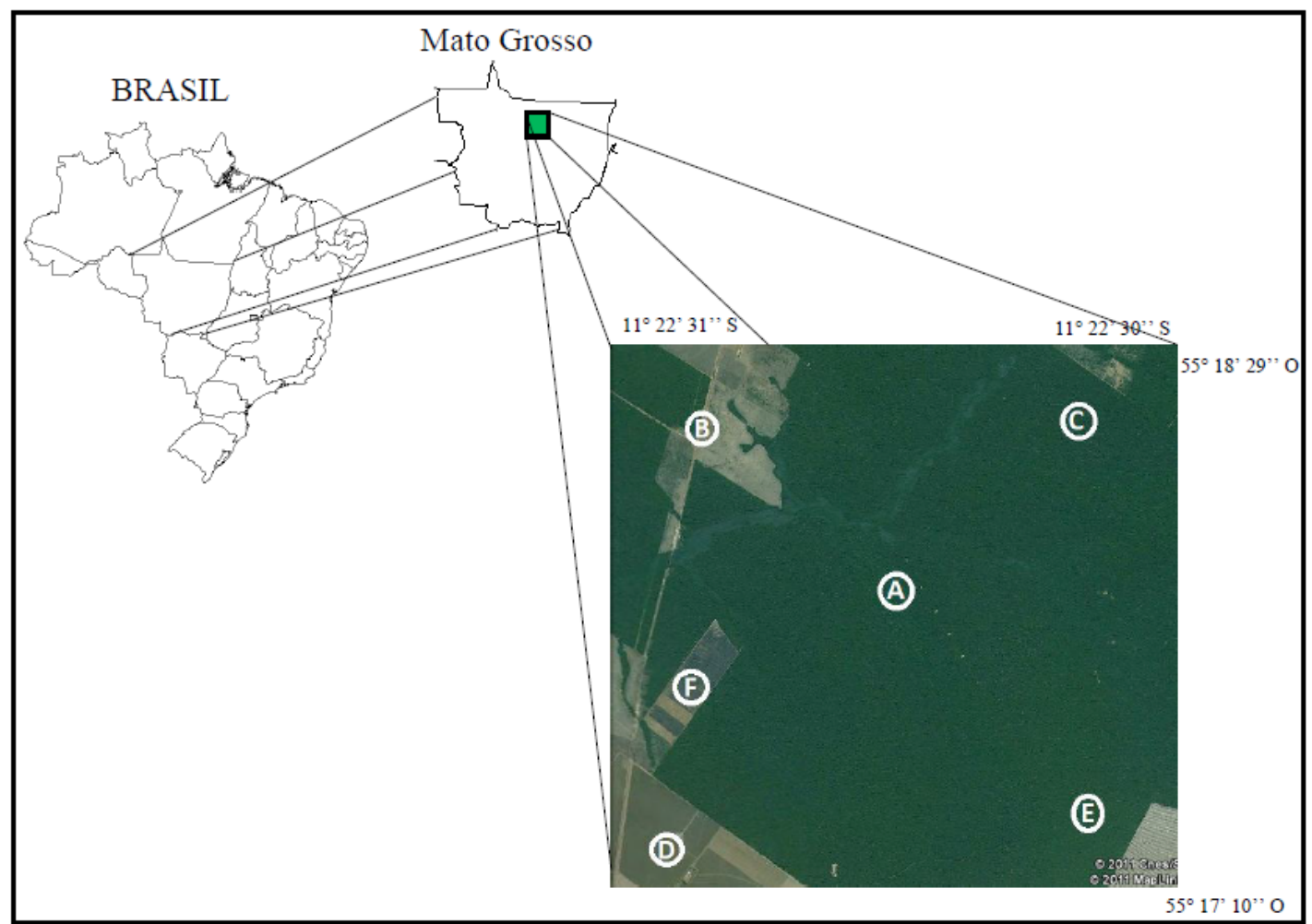

Figura 1- Áreas selecionadas, destacando-se o local de medidas micrometeorológicas (A), áreas de floresta nativa (C) e (E), áreas de cultivo (B), (D) e (F)

O IAF medido em campo teve seus valores mais altos em fevereiro e julho, e menores no mês de junho e início de julho (Tabela 3). Pinto Junior et al. (2011) estimaram o IAF na mesma área de estudo entre 2007 e 2008, e encontraram valor máximo nas estações úmida e transição seca-chuvosa devido ao aumento no crescimento da vegetação da floresta como consequência da maior disponibilidade de água. Esses resultados estão dentro do esperado, pois, estudos recentes com índices de vegetação indicam que a floresta Amazônica apresenta aumento das número de folhas (SALESKA et al., 2007) e maior produtividade primária bruta (BIUDES et al., 2014b) durante a estação seca.

Os valores de IAF estimados pelo produto do MODIS foram menores que os obtidos em campo. O IAF médio da floresta de transição Amazônia-Cerrado estimado pelo produto MODIS durante a estação chuvosa (janeiro a abril) foi de $4,9 \mathrm{~m}^{2} \mathrm{~m}^{-2}$, e durante a estação seca (maio a agosto) foi $5,6 \mathrm{~m}^{2} \mathrm{~m}^{-2}$.

Foi aplicado o teste de normalidade dos resíduos e verificada a distribuição aleatória deles contra os valores de IAF estimados, demonstrando que a regressão e o teste $t$ apresentam-se adequados a esses pressupostos. Os valores do IAF estimados pelo MODIS não apresentaram diferença significativa em contraste com o medido $(p=0,1980)$ com REQM igual $5,51 \mathrm{~m}^{2} \mathrm{~m}^{-2}$, mas foram médias $21,9 \%$ menores que o IAF medido em campo (Figura 2 ) segundo a regressão linear $(\mathrm{p}<0,001)$.

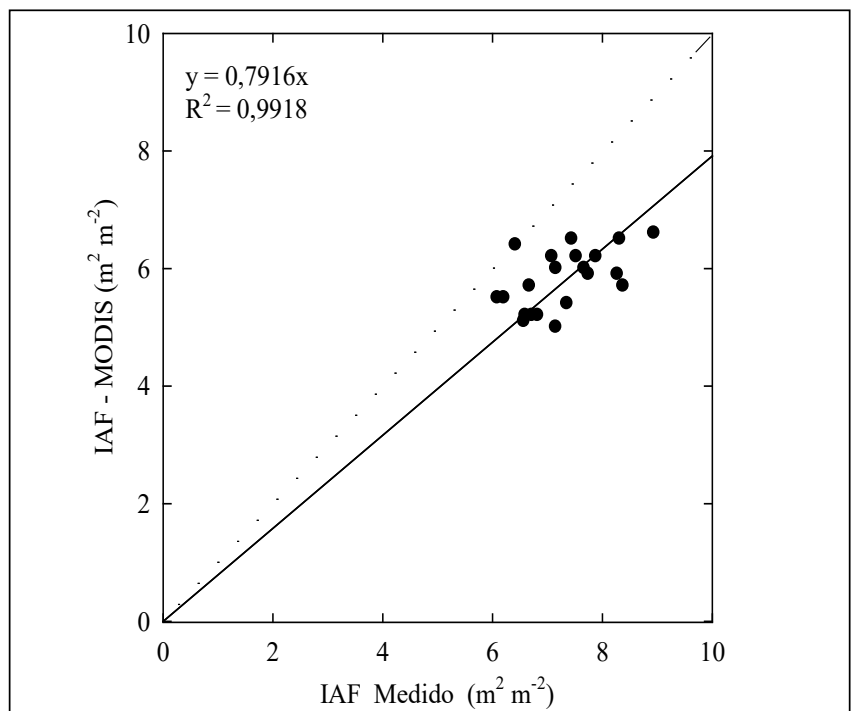

Figura 2- Relação entre o índice de área foliar (IAF) da floresta de transição Amazônia-Cerrado medido em campo e estimado pelo produto MODIS (MOD15A2).

A diferença entre o IAF estimado pelo produto MODIS e o medido em campo ocorreu, provavelmente, devido às influências, tais como efeitos de ruído (caminho atmosférico da radiância), a exposição de sinais assintóticos (saturado) sobre as condições de alta biomassa, e a sensibilidade desse método para variações no fundo do dossel (ARAGÃO et al., 2005).

A interface entre os ecossistemas e a atmosfera é difícil de quantificar devido à variabilidade espacial 


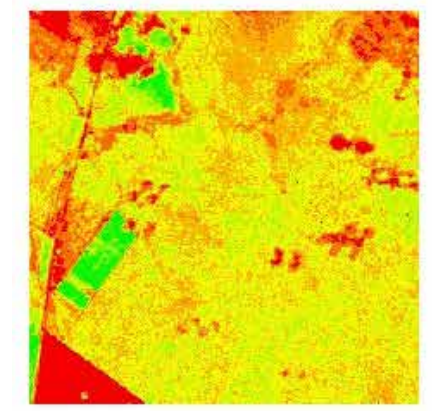

(a) $09-02-2008$

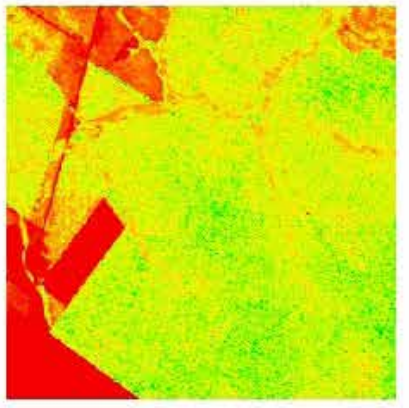

(e) $18-07-2008$

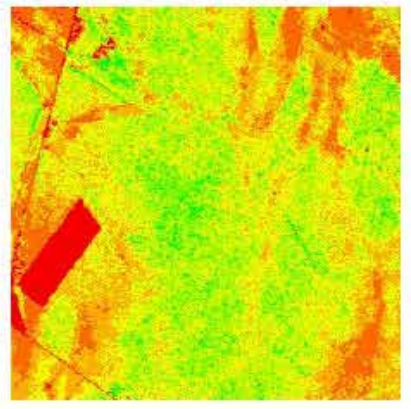

(b) $15-05-2008$

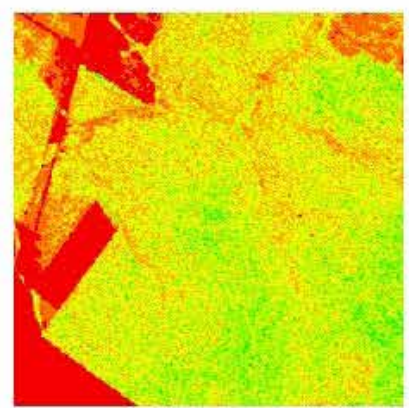

(f) $03-08-2008$

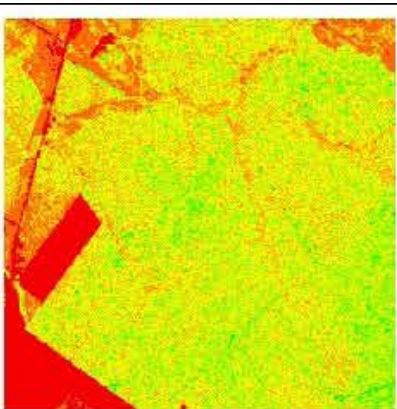

(c) $16-06-2008$

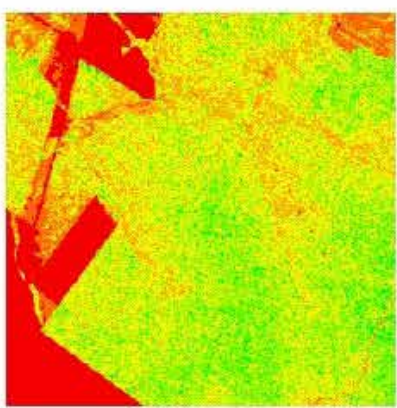

(g) $19-08-2008$

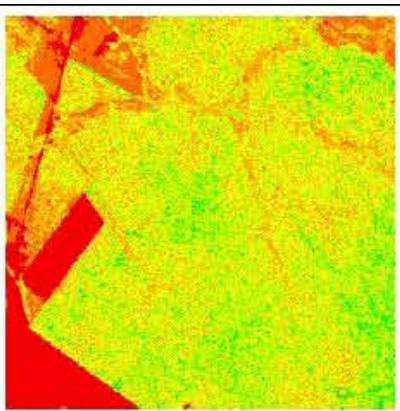

(d) $02-07-2008$

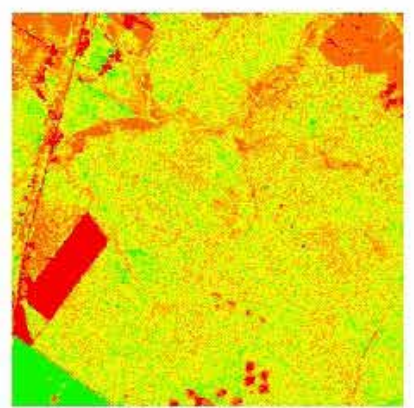

(h) $09-12-2008$

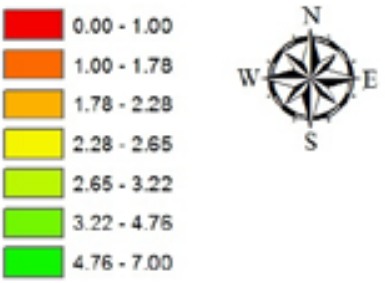

Figura 3 - Variabilidade espacial IAF na área estudada para os dias (a) 02-09-2008, (b) 15-05-2008, (c) 16-06-2008, (d) 02-07-2008, (e) 18-07-2006, (f) 03-08-2008, (g) 19-08-2008, (h) 09-12-2008

(vertical e horizontal) e variabilidade temporal: variações de ciclos anuais e inter-anuais que interagem com padrões estruturais e homogeneidade (BRÉD A, 2003). Fatores como as diferenças de absorção devem ser considerados. Enquanto o algoritmo do MODIS determina o IAF da absorção de radiação pelas folhas somente, $\mathrm{o}$ IAF estimado a partir de dados micrometeorológicos levam em conta a fração de radiação que é transmitida e absorvida pelo dossel (SENNA et al., 2005).

Quanto maior a resolução espacial dos dados do MODIS mais precisa é a derivação da radiação de ondas longas da superfície, pois fornece informações mais detalhadas sobre a atmosfera e a superfície terrestre do que os sensores com uma resolução espacial mais grosseira (WANG \& LIANG , 2009). Devido à resolução espacial de até $1 \mathrm{~km}$, os modelos da superfície da terra utilizando dados MODIS foram aplicados aos ecossistemas naturais. No entanto, a resolução espacial que especifica o tamanho do pixel de imagens de satélite cobrindo a superfície da Terra para o algoritmo do IAF dos dados do MODIS, determinam IAF a $1 \mathrm{~km}$ de resolução espacial, e neste particular o IAF estimado pela observação de campo foi determinado em um único ponto de observação, o que pode explicar em parte as diferenças observadas.

Na tentativa de se estimar o IAF com maior resolução espacial, procedeu-se o seu cálculo pelo METRIC. Apesar das imagens TM - Landast 5 ter resolução temporal de 16 dias, somente foi possível obter 8 cenas. O IAF estimado pelo METRIC apresentou diferença significativa com o IAF medido ( $p=0,000)$, não tendo correlação.

Os resultados obtidos neste trabalho diferiram daqueles obtidos por Allen et al. (2007) e Fideles et al. (2005). A hipótese, nesse caso, para tal diferença, possivelmente resida no fato de que esses autores trabalharam com espécies agrícolas, com padrão de copa definido e uma variabilidade espacial da geometria do dossel inferior à encontrada na floresta de transição.

Mesmo apresentando diferença entre os valores medidos e estimados, foi possível identificar a variabilidade espacial do índice de área foliar. O IAF em toda região variou de 0,01 a $7,0 \mathrm{~m}^{2} \mathrm{~m}^{-2}$ pelo METRIC. Os pontos (B), (F) e (D) da região nas imagens do dia 9 de fevereiro e 9 de dezembro de 2008 (Figuras 3a e 3h) apresentaram maiores valores de IAF, entre 4,0 e $7,0 \mathrm{~m}^{2} \mathrm{~m}^{-2}$ (cores mais 


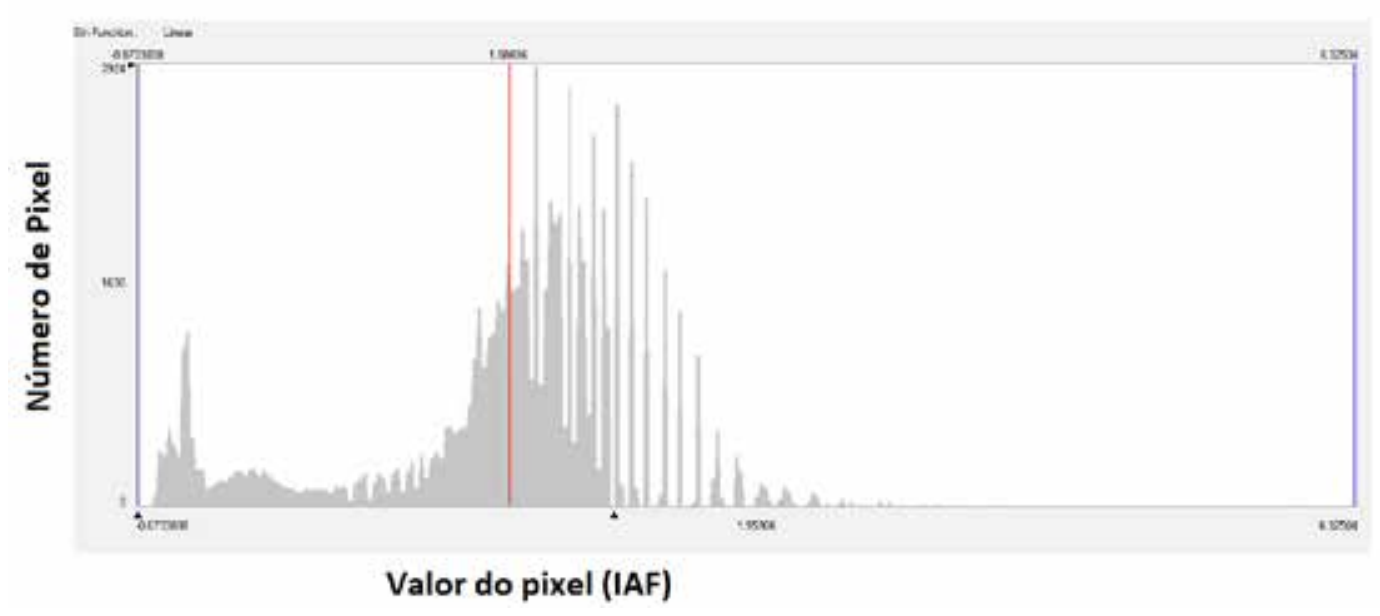

Figura 4 - Histograma do IAF em 03/08/2008, durante a estação seca

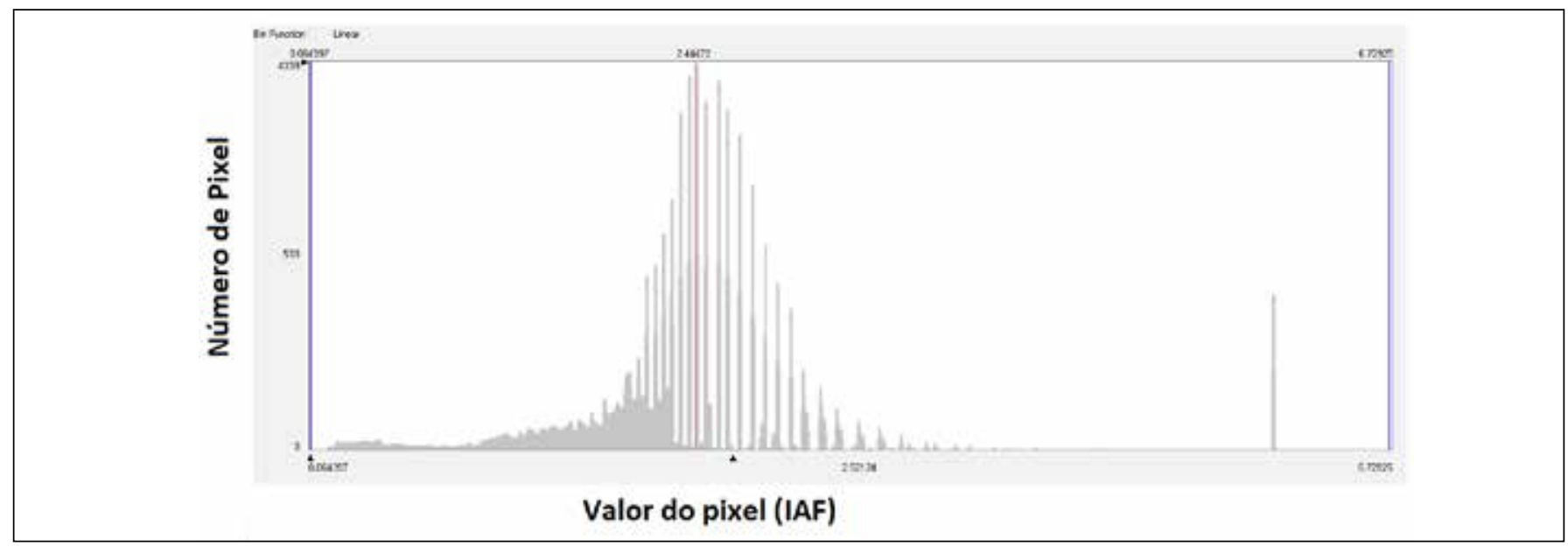

Figura 5 - Histograma do IAF em 09/12/2008, durante a estação chuvosa

claras). Este período corresponde a estação chuvosa de acordo com Priante Filho et al. (2004), o que pode explicar os elevados valores obtidos, refletindo assim o vigor da vegetação.

O IAF da região $F$ apresentou baixos valores, variando de 0,0 a $1,0 \mathrm{~m}^{2} \mathrm{~m}^{-2}$ (cor escura), o que pode ser explicado pela presença de área de pastagem. As regiões D e B apresentam valores de IAF entre 5,0 e 7,0 $\mathrm{m}^{2} \mathrm{~m}^{-2}$ (verde vide escala), no mês de dezembro. Estes elevados valores podem ser explicados pela presença de área de cultivos, ou áreas agrícolas.

Nas Figuras 4 e 5 estão representados os histogramas de frequência do IAF para as estações seca e chuvosa compreendendo os meses de agosto e dezembro. Observa-se, que os maiores valores estão associados à concentração de pontos ao redor da moda de menor frequência, que ficaram para a floresta de transição Amazônia-Cerrado em torno de 3,6 e 4,3 $\mathrm{m}^{2} \mathrm{~m}^{-2}$ nos meses de agosto e dezembro, respectivamente. Em agosto, o IAF médio de toda a cena estudada foi de $1,9 \mathrm{~m}^{2} \mathrm{~m}^{-2}$, enquanto que o IAF em dezembro foi de $2,4 \mathrm{~m}^{2} \mathrm{~m}^{-2}$

Mesmo havendo diferença significativa entre os valores do IAF obtidos pelos métodos analisados, esse aspecto não pode ser conclusivo para não aceitação do METRIC como apropriado para a estimativa dessa variável. Outro aspecto a ser considerado nas diferenças dos valores do IAF é a falta de um maior número de imagens de satélite para a região de estudo e uma calibração apropriada do METRIC para condições de floresta de transição.

\section{Conclusões}

Entre dois métodos que usam dados de sensores orbitais, o produto MODIS apresentou maior aproximação ao IAF medido em campo.

O METRIC não se mostrou capaz de produzir valores de IAF em regiões de transição Amazônia-Cerrado, mas fornece uma boa ideia da variabilidade espacial e dinâmica do IAF na região estudada.

O descarte do METRIC não pode ser conclusivo. Deve-se realizar mais estudos, em diferentes ecossistemas florestais utilizando uma série de dados maior do que a 
utilizada neste trabalho, visando uma nova calibração do modelo para regiões tropicais para confirmar a utilização de ambos os métodos.

\section{Agradecimentos:}

As pesquisas foram mantidas em parte pela Universidade Federal de Mato Grosso (UFMT), Programa de Pós Graduação em Física Ambiental (PPGFA) IF/UFMT, Coordenação de Aperfeiçoamento de Pessoal do Ensino Superior (bolsistas CAPES Proc. nº 9750/13-4 e 9768/130 ), Conselho Nacional de Desenvolvimento Científico e Tecnológico (MCT-CNPq-CT Infra-CT Energia no 07/2006; Code: 620082/2006-2), Fundação de Amparo à Pesquisa do Estado de Mato Grosso (PRONEX/FAPEMAT - 2009 Code: 823971/2009; Edital Universal/FAPEMAT 005/2012 - Proc. no 331763/2012).

\section{Referências}

ALLEN, R., TASUMI, M., TREZZA, R. (2007). Satellitebased energy balance for mapping evapotranspiration with internalized calibration (METRIC) - Model. Journal of Irrigation and Drainage Engineering, 133(395), 380-394.

ARAGÃO, L. E. O. C., SHIMABUKURO, Y .E., ESPÍRITO-SANTO, F. D. B., WILLIAMS, M. (2005). Spatial validation of the collection 4 MODIS LAI product in Eastern Amazonia. IEEE Transactions on Geoscience and Remote Sensing, 43(11), 2526-2534.

BRÉDA, N. J. J. (2003). Ground-based measurements of leaf area index: a review of methods, instruments and current controversies. Journal of Experimental Botany, 54(393),2403-2417.

BIUDES, M.S., VOURLITIS, G. L., MACHADO, N. G., ARRUDA, P.H.Z., NEVES, G.A. R., LOBO, F. A., NEALE, C. M. U., NOGUEIRA, J. S. (2015). Patterns of energy Exchange for tropical ecosystems across a climate gradiente in Mato Grosso, Brazil. Agricultural and Forest Meteorology, 202,112-124.

BIUDES, M.S., MACHADO, N.G., DANELICHEN, V. H. M., SOUZA, M. C., VOURLiTIS, G. L., NOGUEIRA, J.S. (2014a). Ground and remote sensingbased measurements of leaf area index in a transitional forest and seasonal flooded forest in Brazil. International Journal of Biometeorology, 58, 1181-1193.

BIUDES, M. S., SOUZA, M. C., MACHADO, N. G., DANELLICHEN, V. H. M., VOURLITIS, G. L., NOGUEIRA, J. S. (2014b), Modelling gross primary production of a tropical semi-deciduous forest in the southern Amazon Basin. International Journal of Remote Sensing, 35(4), 1540-1562.
CHANDER, G., MARKHAM, B. L., BARSI. J. A. (2007). Revised Landsat-5 Thematic Mapper Radiometric Calibration. IEEE Geoscience and Remote Sensing Letters, 4(3), 490-494.

CROSMAN, E. T., HOREL, J. D. (2009). MODISderived surface temperature of the Great Salt Lake. Remote Sensing of Environment, 113, 73-81.

FIDELES, J. F., NOBREGA, J. Q., RAO, T. V. R., BELTRAO, N. E. M. (2005). Monitoramento de área foliar e biomassa do feijoeiro usando índice de vegetação por diferença normalizada. Revista Brasileira de Agrometeorologia, 13(2), 212-218.

GITELSON, A.A., WARDLOW, B.D., KEYDAN, G. P., LEAVITT, B. (2007). An evaluation of MODIS 250$\mathrm{m}$ data for green LAI estimation in crops. Geophysical Research Letters, 34, L20403.

GOUDRIANN, J. (1988). The bare bones of leafangle distribution in radiation models for canopy photosynthesis and energy exchange. Agricultural and Forest Meteorology, 43, 155-169.

HUETE, A. R. (1988). A soil adjusted vegetation index (SAVI). Remote Sensing of Environment, 25, 295-309.

IQBAL, M. (1983). An Introduction to Solar Radiation. New York: Academic Press.

JUPP, D. L. B., CULVENOR, D. S., LOVELL, J. L., NEWNHAM, G. J., STRAHLER, A. H., WOODCOCK, C. E. (2008). Estimating forest LAI profiles and structural parameters using a ground-based laser called 'Echidna. Tree Physiology, 29, 171-181.

MARKHAM, B. L., BARKER, J. L. (1987). Thematic Mapper bandpass solar exoatmospheric irradiances. International Journal of Remote Sensing, 8(3), 517-523.

MONSI, M., SAEKI, T. (1953). Über den Lichtfaktor in den Pflanzengesellschaften und seine Bedeutung für die Stoffproduckion. Japanese Journal of Botany, 14, 22-52.

MYNENI, R. B., HOFFMAN, S., KNYAZIKHIN, Y., PRIVETTE, J. L., GLASSY, J., TIAN, Y., WANG, Y., SONG, S., ZHANG, Y., SMITH, G. R., LOTSCH, A., FRIELD, M., MORRISETTE, J. T., VOTAVA, P., NEMANI, R. R., RUNNING, S. W. (2002). Global products of vegetation leaf area and fraction absorbed PAR from year one of MODIS data. Remote Sensing Environment, 83, 214-231.

NACKAERTS, K., COPPIN, P., MUYS, B., HERMY, M. (2000). Sampling methodology for LAI measurements with LAI-2000 in small forest stands. Agricultural and Forest Meteorology, 101(4), 247-250. 
PINTO JÚNIOR, O. B., SANCHES, L., LOBO, F. A., BRANDÃO. A. A., NOGUEIRA, J. S. (2011). Leaf area index of a tropical semi-deciduous forest of the southern Amazon Basin. International Journal of Biometeorology, 55, 109-118.

PRIANTE FILHO, N., HAYASHI, M. M. S., NOGUEIRA, J. S., CAMPELO JR, J. H., NUNES, P.C., SANCHES, L., COUTO, E. G., HOEGER, W., RAITER, F., TRIENWEILER, J. L., MIRANDA, E. J., PRIANTE, P. C., PEREIRA, L. C., BIUDES, M. S., FRITZEN, C. L., LACERDA, M., SULI, G. S., SHIRAIWA, S., SILVEIRA, M., VOURLITIS, G. L. (2004). Comparison of the mass and energy exchange of a pasture and a mature transitional tropical forest of the southern Amazon basin during the wet-dry season transition. Global Change Biology, 10, 863-876.

PRIVETTE, J. L., MYNENI, R. B., KNYAZIKHIN, Y., MUKUFUTE, M., ROBERTS, G., TIAN, Y., WANG, Y., LEBLANC, S. G. (2002). Early spatial and temporal validation of MODIS LAI product in Africa. Remote Sensing Environment, 83, 232-243.

RESENDE, S.D.P., LOBO, F. A., DALMAGRO, H.J., BIUDES, M.S., PINTO JÚNIOR, O. B., NOGUEIRA, J. S., VOURLITIS, G. L. (2010). Avaliação de dois métodos para estimativa do índice de área foliar em Floresta de transição Amazônia-Cerrado. Ciência e Natura, 32, 183-195.
SALESKA, S. R., DIDAN, K., HUETE, A. R., DA ROCHA, H. R. (2007). Amazon forests green-up during 2005 drought. Science, 318(5850), 612.

SENNA, M.C.A., COSTA, M.H., SHIMABUKURO, Y. E. (2005). Fraction of photosynthetically active radiation absorbed by Amazon tropical forest: A comparison of field measurements, modeling, and remote sensing. Journal of Geophysical Research, 110, G01008.

VOURLITIS, G. L., LOBO, F. A., ZEILHOFER, P., NOGUEIRA. J. S. (2011). Temporal patterns of net $\mathrm{CO} 2$ exchange for a tropical semideciduous forest of the southern Amazon Basin. Journal of Geophysical Research, 116, G03029.

WANG, W., LIANG, S. (2009). Estimation of highspatial resolution clear-sky longwave downward and net radiation over land surfaces from MODIS data. Remote Sensing of Environment, 113(4), 745-754.

WELLES, J. M. (1990). Some indirect methods of estimating canopy structure. Remote Sensing Reviews, 5, 31-43. 